\title{
Modeling and Control of Physical Processes using Proper Orthogonal Decomposition *
}

\author{
Hung V. Ly ${ }^{1}$ and Hien T. Tran ${ }^{2}$ \\ Center for Research in Scientific Computation-Box 8205 \\ North Carolina State University \\ Raleigh, NC 27695-8205 \\ USA
}

February 12, 1999

\begin{abstract}
Proper orthogonal decomposition (POD) technique (or the Karhunan Loève procedure) has been used to obtain low dimensional dynamical models of many applications in engineering and science. In principle, the idea is to start with an ensemble of data, called snapshots, collected from an experiment or a numerical procedure of a physical system. The POD technique is then used to produce a set of basis functions which spans the snapshot collection. When these basis functions are used in a Galerkin procedure, they yield a finite dimensional dynamical system with the smallest possible degrees of freedom. In this context, it is assumed that the physical system has a mathematical model, which may not be available for many physical and/or industrial applications. In this paper, we consider the steady state Rayleigh-Bénard convection whose mathematical model is assumed to be unknown, but numerical data are available. The aim of the paper is to show that, using the obtained ensemble of data, POD can be used to model accurately the natural convection. Furthermore, this approach is very efficient in the sense that it uses the smallest possible number of parameters and thus is suited for process control. Particularly, we consider two boundary control problems: (a) tracking problem and (b) avoiding hot spot in a certain region of the domain.
\end{abstract}

Keywords. proper orthogonal decomposition, boundary control, Rayleigh-Bénard convection To Appear: Journal of Mathematical and Computer Modeling

\footnotetext{
${ }^{*}$ This research was supported in part by the DOD-MURI grant F49620-95-1-0447 through the Center for Intelligent Design and Manufacturing in Electronics and Materials.

${ }^{1}$ Email: hvly@eos.ncsu.edu.

${ }^{2}$ Email: tran@control.math.ncsu.edu.
} 


\section{Introduction}

There are many families of patterns for which it is possible to obtain a useful systematic characterization. Often, the motivation is that the family is of low dimension, that is, in some sense, any given member of the family might be represented by a small number of parameters. These types of families of patterns occur frequently in both nature and in the literature. Such examples include turbulent flows [1, 2, 3, 4], image processing [5, 6], data compression [7], human speech [8], and human faces [9]. The technique applied in these applications is known as the proper orthogonal decomposition (POD), although it also goes by other names such as the Karhunen-Loève decomposition [10], principal component analysis [11], and the Hotelling transform [12].

POD seems to have been first proposed [13] in 1901 and then again [14] in 1933. Since its introduction, the method has received much attention as a tool to analyze complex physical systems. One such important application was the attraction of spatial organized motions in fluid flows. Theodorsen [15] and later Townsend [16] observed and indicated that there are large-scale organized motions embedded in turbulent shear flows. Lumley [1] used POD as an unbiased way for extracting structures from turbulent flows. Other applications of POD include channel flows $[17,18]$, square-duct flows [19], and shear flows [20, 21]. Other scientists have also applied the POD technique to fluid related problems. For instance, POD has been applied to the Burgers' equation [22], the Ginzburg-Landau equation and the Bénard convection [23]. Ly and Tran [24] have used POD to simulate and solve an optimal control problem for fluid flows in a horizontal chemical vapor deposition reactor. In addition, Banks, del Rosario and Smith [25] have applied POD to design feedback control for a thin shell model. The goal of the approach is to represent an ensemble of data (called snapshots), obtained from physical experiments or from detailed numerical simulations, in terms of an optimal coordinate system. That is, the snapshots can be generated by a smallest possible set of basis functions. Among the optimality properties is the fact that, used in conjunction with the Galerkin method, the POD yields an optimal set of basis functions in the sense that the resulting truncated system of ODE's captures the maximum amount of kinetic energy among all possible truncations of the same order.

In general, to implement numerical methods such as POD procedure used together with the Galerkin method, finite element, finite volume, or spectral method, for applications in engineering or science it is assumed that the physical system can be described by a mathematical model (in general, a nonlinear distributed parameter system with boundary/initial conditions). The governing equations are usually derived based on some simplified assumptions about the physical system. On the other hand, many applications in engineering or science are complex enough that a mathematical description is either not possible or when a model is obtained it can't be implemented without undue complications. For example, the degree of freedom of distributed parameter systems is essentially infinite and the relevant mathematical theory is either too complicated or is still lacking (in the case of nonlinear distributed parameter system) to be implemented in an industry. In this study, we consider a physical process, whose mathematical model is assumed not to be available, but data can be collected from the system. For example, in a physical setting, data can be collected from thermal images or from thermocouples. Our primary goal is to show that the characteristic features, extracted from the collected data using the POD procedure, can be used to model the physical system efficiently and accurately. Moreover, due the optimality of the POD technique, this approach guarantees that the number of parameters used to represent the physical process is smallest possible. Consequently, process control for such system becomes apparent. For our physical application, we consider the Rayleigh-Bénard convection problem. Here, the governing equations are used only for the purpose of generating an ensemble of data. The classical Rayleigh-Bénard problem offers a first approach to highly complicated convection flow. Natural convection is both a challenging and an interesting subject due to the coupling of the fluid flow and the energy transport. It has been extensively studied in the literature because of its importance to many engineering applications and because it is often found to be the controlling mechanism in many natural occuring processes. The numerous applications are found, for instance, in meteorology, geophysics, crystal growth in microprocessor manufacturing, and building heat transfer. An excellent overview of this subject can be found 
in $[26]$.

More specifically, we consider in this paper a mixed convection in a square cavity heated on one wall and cooled on the opposite wall. It is well known that in such a configuration, for moderate Rayleigh number, the fluid flow is characterized by a single large cell; the fluid ascends along the hot wall, descends along the cold wall, and turns at the opposite end of the cavity. For small Rayleigh numbers the flow is weak and heat is transferred by conduction across the fluid. However, with increasing Rayleigh number this flow becomes unstable (see, e.g., [27]). As noted above, thermal convection is the result of the coupling between the fluid flow and heat transport. However, in this study, the collected ensemble of data consists of only partial information, namely, the thermal data. Using this ensemble of thermal numerical data, the POD expansion produces an "optimal" compressed description of that ensemble. This description is a truncated series expansion in terms of a set of basis functions. As an evaluation of the success of the procedure, we project data from outside of the set of snapshots onto the set of optimal basis functions. Even in this case, the relative errors were remarkably good (less than $3 \%$ ). In addition, we also show that the resulting eigenfunctions and coefficients associated with each eigenfunction can be used to solve effectively two boundary control problems. One application is the tracking problem of finding the thermal boundary condition so that the isotherms inside the cavity match with a prescribed temperature distribution. The other problem is to determine the hot wall temperature so that certain region inside the cavity stayed below a given temperature. These studies offer an important first step to modeling and real-time control of complex physical systems.

The organization of the paper is as follow. We describe in $\S 2$ the proper orthogonal decomposition procedure. The physical problem and snapshot construction are described in $\S 3$. Section 4 describes the computation of the POD basis functions, their uses in modeling convective motion, and error analysis. Finally, applications to two practical boundary control problems are discussed in $\S 5$.

\section{The Proper Orthogonal Decomposition}

Let $U_{i}(\overrightarrow{\mathbf{x}}), i=1,2, \ldots, N$ denote the set of $N$ observations (also called snapshots) of some physical process taken at position $\overrightarrow{\mathbf{x}}=(x, y)$. The average of the ensemble of snapshots is given by

$$
\bar{U}=<U>=\frac{1}{N} \sum_{i=1}^{N} U_{i}(\overrightarrow{\mathbf{x}})
$$

We form new ensemble by focusing on deviations from the mean as follows:

$$
V_{i}=U_{i}-\bar{U}
$$

We wish to find an optimal compressed description of the sequence of data (2.2). One description of the process is a series expansion in terms of a set of basis functions. Intuitively, the basis functions should in some sense be representative of the members of the ensemble. Such a coordinate system, which possesses several optimality properties (to be discussed in the sequel), is provided by the Karhunen-Loève expansion, where the basis functions $\mathbf{\Phi}$ are, in fact, admixtures of the snapshots and are given by:

$$
\mathbf{\Phi}=\sum_{i=1}^{N} a_{i} V_{i}(\overrightarrow{\mathbf{x}}) .
$$

Here, the coefficients $a_{i}$ are to be determined so that $\boldsymbol{\Phi}$ given by (2.3) will most resembles the ensemble $\left\{V_{i}(\overrightarrow{\mathbf{x}})\right\}_{i=1}^{N}$. More specifically, we look for a function $\boldsymbol{\Phi}$ to maximize

$$
\frac{1}{N} \sum_{i=1}^{N}\left|\left(V_{i}, \boldsymbol{\Phi}\right)\right|^{2},
$$


subject to

$$
(\boldsymbol{\Phi}, \mathbf{\Phi})=\|\mathbf{\Phi}\|^{2}=1,
$$

where $(\cdot, \cdot)$ and $\|\cdot\|$ denote the usual $\mathbf{L}^{2}$ inner product and $\mathbf{L}^{2}$-norm, respectively.

It follows that (see, e.g., [24]) the basis functions are the eigenfunctions of the integral equation

$$
\int \mathbf{C}\left(\overrightarrow{\mathbf{x}}, \overrightarrow{\mathbf{x}}^{\prime}\right) \boldsymbol{\Phi}\left(\overrightarrow{\mathbf{x}}^{\prime}\right) d \overrightarrow{\mathbf{x}}^{\prime}=\lambda \boldsymbol{\Phi}(\overrightarrow{\mathbf{x}})
$$

where the kernel is given by

$$
\mathbf{C}\left(\overrightarrow{\mathbf{x}}, \overrightarrow{\mathbf{x}}^{\prime}\right)=\frac{1}{N} \sum_{i=1}^{N} V_{i}(\overrightarrow{\mathbf{x}}) V_{i}\left(\overrightarrow{\mathbf{x}}^{\prime}\right)
$$

Substituting (2.3) into (2.5) yields the eigenvalue problem:

$$
\sum_{j=1}^{N} \mathbf{L}_{i j} a_{j}=\lambda a_{i}
$$

where $\mathbf{L}_{i j}=\frac{1}{N}\left(V_{i}, V_{j}\right)$ is a symmetric and nonnegative matrix. Thus, we see that our problem amounts to solving for the eigenvectors of an $N \times N$ matrix where $N$ is the size of the ensemble of snapshots. Straightforward calculation (see also [24]) shows that the cost functional

$$
\frac{1}{N} \sum_{i=1}^{N}\left|\left(V_{i}, \boldsymbol{\Phi}\right)\right|^{2}=(\lambda \boldsymbol{\Phi}, \boldsymbol{\Phi})=\lambda .
$$

which is maximized when the coefficients $a_{i}$ 's of (2.3) are the elements of the eigenvector corresponding to the largest eigenvalue of $\mathbf{L}$.

Remarks. Alternatively, we can consider the discrete Karhunen-Loève expansion to find an optimal representation of the ensemble of snapshots. In the two-dimensional case, each sample of snapshots $U_{i}(x, y)$ (defined on a set of $n \times n$ nodal points $(x, y)$ ) can be expressed as an $n^{2}$ dimensional vector $\overrightarrow{\mathbf{u}}_{i}$ as follows:

$$
\overrightarrow{\mathbf{u}}_{i}=\left[\begin{array}{l}
\overrightarrow{\mathbf{u}}_{i 1} \\
\overrightarrow{\mathbf{u}}_{i 2} \\
\vdots \\
\overrightarrow{\mathbf{u}}_{i j} \\
\vdots \\
\overrightarrow{\mathbf{u}}_{i n^{2}}
\end{array}\right]
$$

where $\overrightarrow{\mathbf{u}}_{i j}$ denotes the $j$ th component of the vector $\overrightarrow{\mathbf{u}}_{i}$. Here, the discrete covariance matrix of the ensemble $\overrightarrow{\mathbf{u}}$ is defined as [12]:

$$
C_{\overrightarrow{\mathbf{u}}}=E\left\{\left(\overrightarrow{\mathbf{u}}-\overrightarrow{\mathbf{m}}_{\overrightarrow{\mathbf{u}}}\right)\left(\overrightarrow{\mathbf{u}}-\overrightarrow{\mathbf{m}}_{\overrightarrow{\mathbf{u}}}\right)^{T}\right\},
$$

where

$$
\overrightarrow{\mathbf{m}}_{\overrightarrow{\mathbf{u}}}=E\{\overrightarrow{\mathbf{u}}\}
$$

is the mean vector, $E$ is the expected value. Equations (2.9) and (2.10) can be replaced by:

$$
C_{\overrightarrow{\mathbf{u}}}=\frac{1}{N}\left[\sum_{i=1}^{N} \overrightarrow{\mathbf{u}}_{i} \overrightarrow{\mathbf{u}}_{i}^{T}\right]-\overrightarrow{\mathbf{m}}_{\overrightarrow{\mathbf{u}}} \overrightarrow{\mathbf{m}}_{\overrightarrow{\mathbf{u}}}^{T}
$$

and

$$
\overrightarrow{\mathbf{m}}_{\overrightarrow{\mathbf{u}}}=\frac{1}{N} \sum_{i=1}^{N} \overrightarrow{\mathbf{u}}_{i}
$$

respectively. The size of the covariance matrix is now of dimension $n^{2} \times n^{2}$, where $n^{2}$ is the number of nodal points (or pixels of the samples). The eigenvector corresponding to the largest eigenvalue of $C_{\overrightarrow{\mathbf{u}}}$ is the dominant POD mode. However, this approach is inhibitely expensive, especially when the samples are of higher dimensions, because one has to solve a very large eigenvalue problem. 


\section{Rayleigh-Bénard Convection and Snapshots' Construc- tion}

In this section, we consider the steady state problem of Rayleigh-Bénard system in a square cavity. It is well known that if the Rayleigh number exceeds a certain critical value, buoyancydriven thermal cellular convection, combined with an increase in the heat flux through the fluid layer, will begin. The mathematical formulation described below is used solely for the purpose of generating the numerical data (snapshots) to be used in the POD procedure.

\subsection{Rayleigh-Bénard Convection}

Let $\Omega$ be the rectangular cavity $\{(x, y) \mid 0 \leq x \leq L, 0 \leq y \leq L\}$ in $\mathbb{R}^{2}$ which is filled with fluid. The top, $\Gamma_{\text {top }}$, and bottom, $\Gamma_{\text {bot }}$, walls are insulated, the left wall, $\Gamma_{\text {left }}$, is maintained at a constant cold temperature $T_{0}$ while the right wall, $\Gamma_{\text {right }}$, is heated with a parabolic profile $\frac{4\left(T_{1}-T_{0}\right)}{L^{2}} y(L-y)+T_{0}$, where $T_{1}>T_{0}$ (see Figure 1$)$.

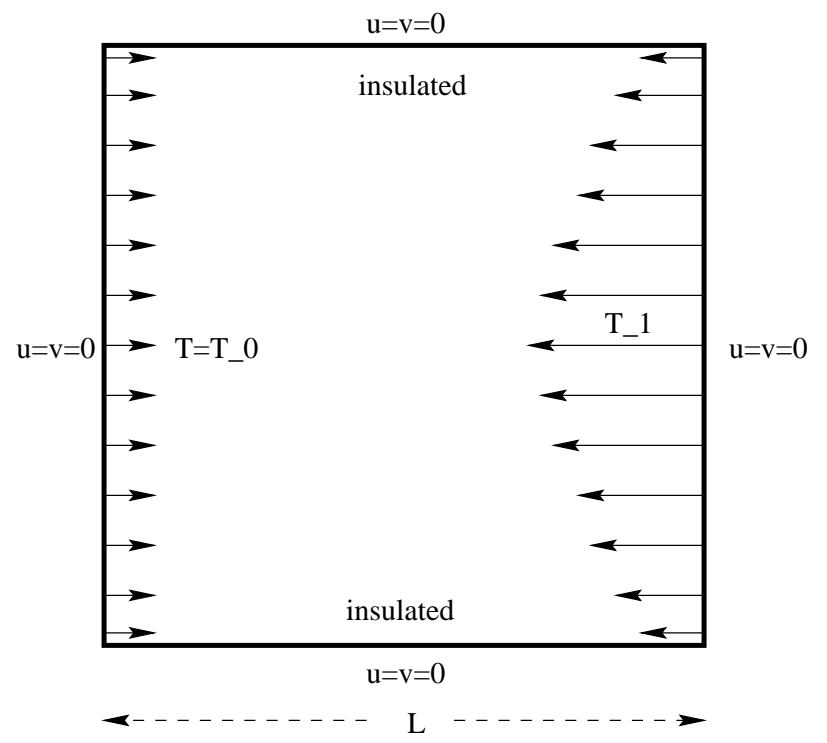

Figure 1: Dimensionalized Problem Definition of Rayleigh-Bénard Convection

We assume that the fluid is viscous, incompressible and that the flow is laminar. Under these assumptions, the governing equations for the system are given by (see, e.g., [26]):

Continuity:

$$
\nabla \cdot \overrightarrow{\mathbf{u}}=0
$$

Momentum:

$$
\rho \frac{\partial \overrightarrow{\mathbf{u}}}{\partial t}+\rho \overrightarrow{\mathbf{u}} \cdot \nabla \overrightarrow{\mathbf{u}}=-\nabla P+\rho g \vec{j} \beta\left(T-T_{0}\right)+\mu \nabla^{2} \overrightarrow{\mathbf{u}},
$$

Energy:

$$
\rho c_{p} \frac{\partial T}{\partial t}+\rho c_{p} \overrightarrow{\mathbf{u}} \cdot \nabla T=\kappa \nabla^{2} T,
$$

where $\overrightarrow{\mathbf{u}}=[u, v]^{T}, T$ and $p$ represent the fluid velocity, temperature and pressure respectively; $\mu$ stands for the dynamic viscosity, $\kappa$ the thermal conductivity, $c_{p}$ the specific heat, $\rho$ the density, $\beta$ the volume expansion constant, $g$ the gravity and $\vec{j}$ the vertical unit vector.

The hydrodynamic and thermal boundary conditions on the fixed surfaces are: 
- At the top and bottom walls of the cavity

$$
\begin{gathered}
u=v=0 \\
\frac{\partial T}{\partial \vec{n}}=0,
\end{gathered}
$$

- At the left wall

$$
\begin{gathered}
u=v=0 \\
T=T_{0}
\end{gathered}
$$

- At the right wall

$$
\begin{gathered}
u=v=0 \\
T=\frac{4\left(T_{1}-T_{0}\right)}{L^{2}} y(L-y)+T_{0},
\end{gathered}
$$

The initial conditions on $\Omega$ are:

$$
\begin{aligned}
\overrightarrow{\mathbf{u}}(x, y, 0) & =\overrightarrow{\mathbf{u}}^{0}(x, y) \\
T(x, y, 0) & =T^{0}(x, y) .
\end{aligned}
$$

We next rescale the equations (3.1)-(3.3) to obtain the dimensionless forms. Let's denote by

$$
R a=\frac{c_{p} \rho^{2} \beta g\left(T_{1}-T_{0}\right) L^{3}}{\mu \kappa}
$$

the Rayleigh number and

$$
\operatorname{Pr}=\frac{\mu c_{p}}{\kappa}
$$

the Prandtl number. We now substitute the following relations

$$
\begin{aligned}
\overrightarrow{\mathbf{u}} & =\frac{\kappa \sqrt{R a P r}}{\rho c_{p} L} \tilde{\overrightarrow{\mathbf{u}}} & T & =\left(T_{s}-T_{0}\right) \tilde{T}+T_{0} \\
P & =\frac{\mu \kappa \sqrt{R a P r}}{\rho c_{p} L^{2}} \tilde{P} & t & =\frac{\rho c_{p} L^{2}}{\kappa \sqrt{R a P r}} \tilde{t} \\
x & =L \tilde{x} & y & =L \tilde{y}
\end{aligned}
$$

in equations (3.1)-(3.3) and in the boundary and initial conditions (3.4)-(3.7), then rename the variables $[\tilde{\overrightarrow{\mathbf{u}}}, \tilde{T}, \tilde{P}, \tilde{x}, \tilde{y}, \tilde{t}]$ as $[\overrightarrow{\mathbf{u}}, T, P, x, y, t]$ respectively, to obtain the following nondimensionalized system of governing equations:

$$
\begin{gathered}
\nabla \cdot \overrightarrow{\mathbf{u}}=0, \\
\gamma \frac{\partial \overrightarrow{\mathbf{u}}}{\partial t}+\gamma \overrightarrow{\mathbf{u}} \cdot \nabla \overrightarrow{\mathbf{u}}=-\nabla P+\gamma \vec{j} T+\nabla^{2} \overrightarrow{\mathbf{u}}, \\
\gamma \frac{\partial T}{\partial t}+\gamma \overrightarrow{\mathbf{u}} \cdot \nabla T=\nabla^{2} T, \\
u=v=\frac{\partial T}{\partial \vec{n}}=0 \quad \text { on } \Gamma_{\text {top }} \text { and } \Gamma_{\mathrm{bot}}, \\
u=v=0, \quad T=0 \quad \text { on } \Gamma_{\text {left }}, \\
u=v=0, \quad T=4 y(1-y) \quad \text { on } \Gamma_{\text {right }},
\end{gathered}
$$




$$
\begin{gathered}
\overrightarrow{\mathbf{u}}(x, y, 0)=\frac{\rho c_{p} L}{\kappa \sqrt{\operatorname{RaPr}}} \overrightarrow{\mathbf{u}}^{0}(x, y) \\
T(x, y, 0)=\frac{1}{T_{1}-T_{0}}\left(T^{0}(x, y)-T_{0}\right),
\end{gathered}
$$

where $\gamma=\sqrt{\frac{R a}{P r}}$. The domain now becomes a unit square cavity. Consequently, the steady-state system of governing equations are given by (see Figure 2):

$$
\begin{gathered}
\nabla \cdot \overrightarrow{\mathbf{u}}=0, \\
\gamma \overrightarrow{\mathbf{u}} \cdot \nabla \overrightarrow{\mathbf{u}}=-\nabla P+\gamma \vec{j} T+\nabla^{2} \overrightarrow{\mathbf{u}}, \\
\gamma \overrightarrow{\mathbf{u}} \cdot \nabla T=\nabla^{2} T, \\
u=v=\frac{\partial T}{\partial \vec{n}}=0 \quad \text { on } \Gamma_{\text {top }} \text { and } \Gamma_{\mathrm{bot}}, \\
u=v=0, \quad T=0 \quad \text { on } \Gamma_{\text {left }}, \\
u=v=0, \quad T=4 y(1-y) \quad \text { on } \Gamma_{\text {right }} .
\end{gathered}
$$

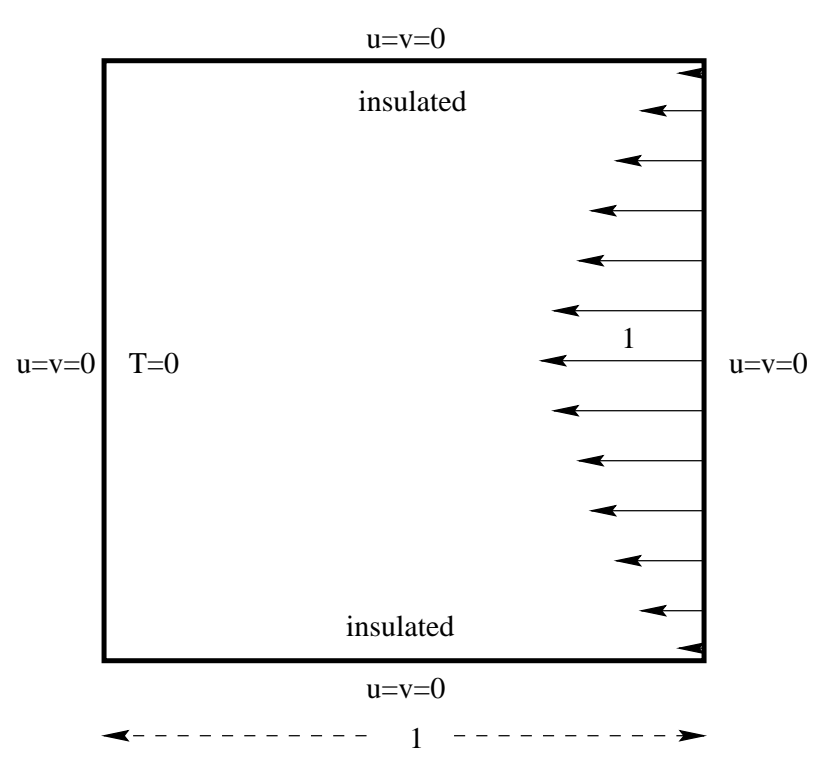

Figure 2: Non-dimensionalized Problem Definition of Rayleigh-Bénard Convection

\subsection{Construction of Snapshots}

We assume that the temperature on the left-hand-side of the cavity is cold at $300^{\circ} K$ and the fluid has the following properties:

$$
\begin{array}{ll}
c_{p} \equiv 5.24 \times 10^{3} \frac{J}{\mathrm{kgK}} & \mu \equiv 97 \times 10^{-6} \frac{\mathrm{J}}{\mathrm{kgK}} \\
\rho \equiv 1.0 \frac{\mathrm{kg}}{\mathrm{m}^{3}} & \kappa \equiv 580 \times 10^{-3} \frac{\mathrm{W}}{\mathrm{mK}} \\
\beta \equiv 1010 \times 10^{-6} \frac{1}{K} & L \equiv 0.10 \mathrm{~m},
\end{array}
$$

Then for each heat intensity $T_{1}$ applied to the right wall of the cavity, there are two corresponding dimensionless numbers, the Prandtl number $\operatorname{Pr}$ and the Rayleigh number $R a$. And for the above parameters, these constants have values:

$$
\operatorname{Pr}=\frac{\mu c_{p}}{\kappa}=0.876
$$


and

$$
R a=\frac{c_{p} \rho^{2} \beta g L^{3}}{\mu \kappa}\left(T_{1}-300\right)=921.9\left(T_{1}-300\right) .
$$

It is well known [26] that when the Rayleigh number $R a$ (and equivalently $T_{1}$ ) exceeds a certain critical value, buoyancy driven thermal convection cells will begin to form in the cavity. In this paper, we restrict our flow to laminar regime and, consequently, we restrict Rayleigh numbers to be less than $10^{6}$. This corresponds to values of the right wall temperature $T_{1}$ in the range from $300^{\circ} \mathrm{K}$ to $1385^{\circ} \mathrm{K}$.

The ensemble of snapshots (see Figure 3) used in this paper was obtained numerically by utilizing a commercial fluid dynamics package called FIDAP version 7.6. The steady-state system of non-dimensionalized equations (3.15)-(3.20) was discretized using 81 quadrilateral (9-nodal quadratic) elements. The resulting discrete nonlinear equations were solved for the velocity fields, temperature distributions, and pressure using values of Rayleigh numbers, $R a$, equal to $1,000,5,000$, and from $10,000 \ldots 200,000$ in increments of 10,000 . The corresponding values of the right wall temperature, $T_{1}$, ranges from $301.08^{\circ} \mathrm{K}$ to $516.94^{\mathrm{o}} \mathrm{K}$. Only 22 snapshots of temperature distributions were used in the POD procedure. In addition, we generate four extra temperature snapshots corresponding to $R a$ equals to $500,71,250,115,000$, and 250,000 for reasons to be discussed later.
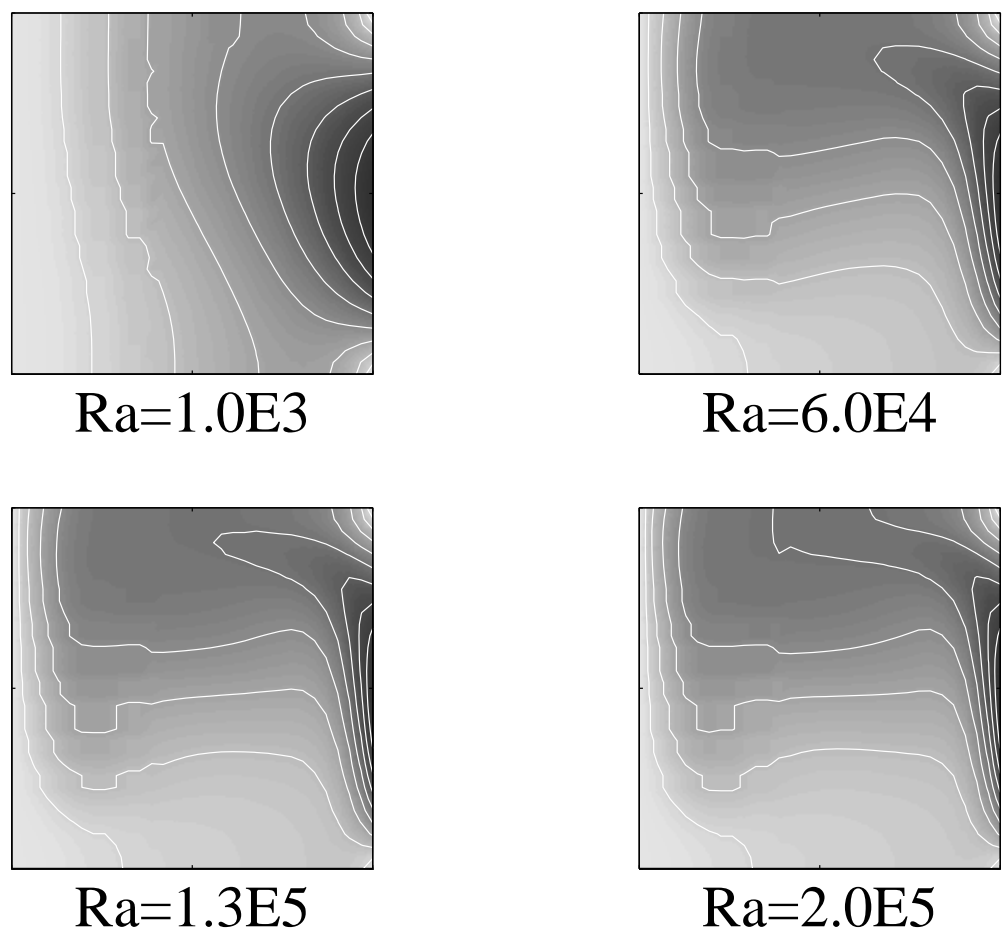

Figure 3: A sample of Calculated Snapshots of Temperature Distribution

\section{Construction of POD Basis Functions}

Let $U_{i}^{R a}(\overrightarrow{\mathbf{x}}), i=1,2, \ldots, 22$ denote the set of 22 temperature snapshots as described in $\S 3.2$ above. Here, we use the superscript $R a$ to denote the dependency of the snapshots on the Rayleigh number. For convenience in notation, the superscript is sometimes omitted in the 
following discussion. The average of the ensemble of snapshots is given by

$$
\bar{U}=<U>=\frac{1}{22} \sum_{i=1}^{22} U_{i}^{R a}(\overrightarrow{\mathbf{x}}) .
$$

We form new ensemble by focusing on deviations from the mean as follows:

$$
V_{i}=U_{i}-\bar{U}, \quad i=1,2, \ldots, 22 .
$$

We then apply the POD procedure to fluctuations of the temperature from the mean, $V_{i}$ 's. The coefficients $a_{i}$ 's of the POD basis functions $\boldsymbol{\Phi}_{k}$, defined by (2.3), are the entries of the $k$ th eigenvectors corresponding to the $k$ th largest eigenvalue of the $N \times N$ covariance matrix $\mathbf{L}$ (see $\S 2)$ :

$$
\mathbf{L}_{i j}=\frac{1}{22} \int_{\Omega} V_{i}(\overrightarrow{\mathbf{x}}) V_{j}(\overrightarrow{\mathbf{x}}) d \overrightarrow{\mathbf{x}} .
$$

Since the covariance matrix is a symmetric and nonnegative matrix, the eigenvalues, $\lambda_{k}$ are real and nonnegative. We arrange the eigenvalues in decreasing order as $\lambda_{1} \geq \lambda_{2} \geq \cdots \geq \lambda_{22} \geq 0$. Thus $\boldsymbol{\Phi}_{1}$ is the basis function corresponding to the largest eigenvalue. A representative of these basis functions is given in Figure 4.

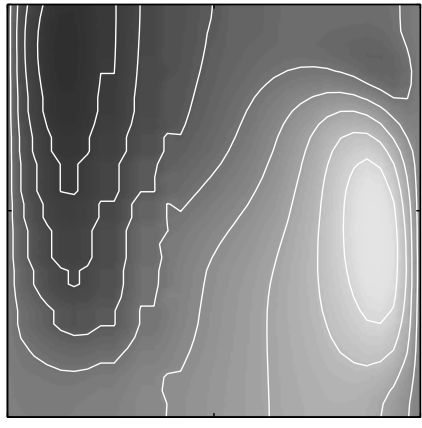

POD Basis \#1

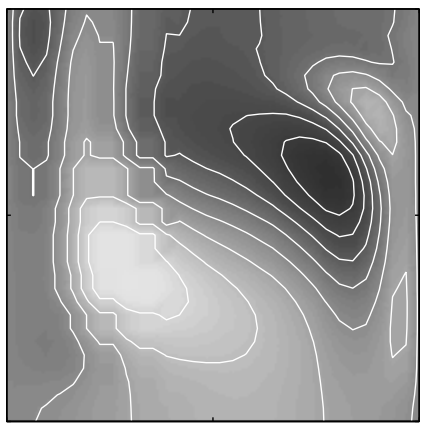

POD Basis \#3

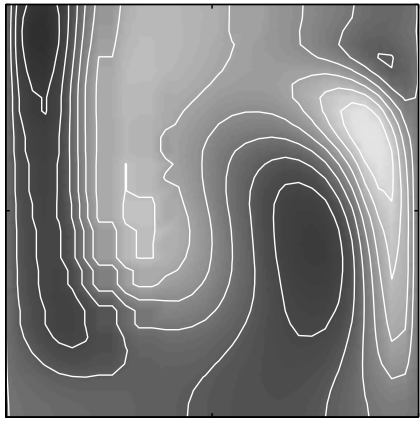

POD Basis \#2

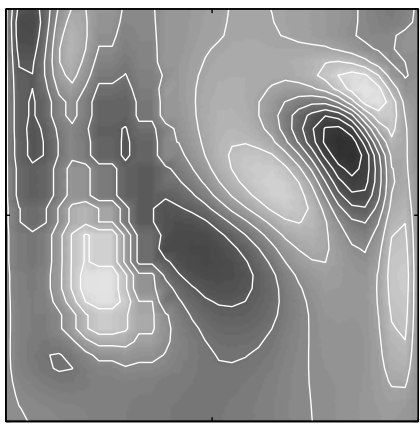

POD Basis \#4

Figure 4: A sample of Calculated POD Basis Functions

Any snapshot in the ensemble can be represented as a linear combination of the basis functions as follows:

$$
U^{R a}=\bar{U}+\sum_{k=1}^{N} \alpha_{k}^{R a} \boldsymbol{\Phi}_{k}
$$


where

$$
\alpha_{k}^{R a}=\left(\mathbf{\Phi}_{k}, U^{R a}-\bar{U}\right) .
$$

and $N=22$.

We next investigate at how much error is introduced by truncating the series (4.3). That is, we now consider the approximation

$$
U_{M}^{R a} \approx \bar{U}+\sum_{k=1}^{M} \alpha_{k}^{R a} \boldsymbol{\Phi}_{k}
$$

where $M \ll N(=22)$. It is well known that (see, e.g., [24]) the expansion (4.5) is optimal. In particular, among all linear combinations, the POD is the most efficient, in the sense that, for a given number of modes $M$, the POD decomposition will capture the most possible kinetic energy. In fact, one way to quantify the error of the approximation is by computing the relative error as:

$$
E^{M}=\frac{\left\|U-U_{M}^{R a}\right\|}{\|U\|}
$$

Figure 5 shows the relative errors of all 22 snapshots against reconstructed solutions using $M$ POD modes where $M=1,2,3$, and 4 . We note that for $M=3$ the relative error is already less than $0.5 \%$.

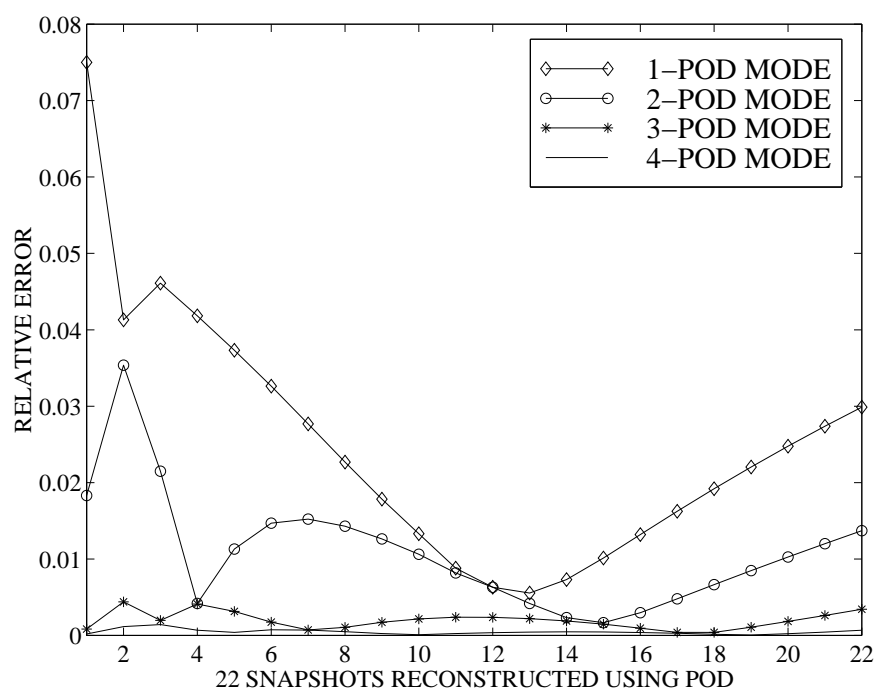

Figure 5: Relative Errors of Each Snapshots Plotted for Various Values of $M$

The crucial question is, therefore, how to choose $M$, a priori, so that (4.5) is a good approximation to (4.3). As already indicated in several publications (see, e.g., [24]), $\sum_{i=1}^{M} \lambda_{i}$, where $\lambda_{i}$ denotes the $i$ th eigenvalue of the covariance matrix $\mathbf{L}$, represents the average kinetic energy contained in the first $M$ modes. Hence, to capture most of the energy of the system contained in the $N$ POD elements, it suffices to choose $M$ so that $\sum_{i=1}^{M} \lambda_{i} \approx \sum_{i=1}^{N} \lambda_{i}$. In fact, the ratio $\sum_{i=1}^{M} \lambda_{i} / \sum_{i=1}^{N} \lambda_{i}$ yields the percentage of the total kinetic energy in the $N$ POD elements that is contained in the first $M$ POD elements. Since the eigenvalues are in descending order, $\lambda_{1} \geq \lambda_{2} \geq \cdots \geq \lambda_{N} \geq 0$, one can reasonably expect to achieve a high percentage of the total kinetic energy with $M$ sufficiently smaller than $N$. In our case, for an ensemble of 22 snapshots, an approximation with $M=1$ yielded a ratio of .9334 . However, when $M=3$, this ratio increases to 0.9996 resulting in a truly significant computational efficiency.

To be useful in developing both open loop and feedback control strategies, we now demonstrate that by using the same POD basis functions, we are able to model the Rayleigh-Bénard 
system for Rayleigh number equals to $500,71,250$, and 250,000 . We note that the values 500 and 250,000 are not in the range used orginally to compute the ensemble of 22 snapshots. To compute the POD approximation for each new Rayleigh number, we fit, for each mode $k$, the original Rayleigh dependent coefficients $\alpha_{k}^{R a}$, by a cubic spline interpolation. We then evaluate the cubic spline polynomial at $R a=500,71,250$, and 250,000 to obtain the corresponding coefficients $\bar{\alpha}_{k}^{R a}$ 's. The approximations, as computed by the POD expansion using $M=3$, are compared against the FIDAP solutions in Figure 6. The plots show a remarkably good agreement. Also Figure 7 shows the relative errors plotted against the number of POD modes $M$. For $M=3$ the maximum relative error is already less than $3 \%$. The approximation, $\bar{U}+\sum_{k=1}^{3} \bar{\alpha}_{k}^{R a} \mathbf{\Phi}_{k}$ is then used to compute the POD solutions.

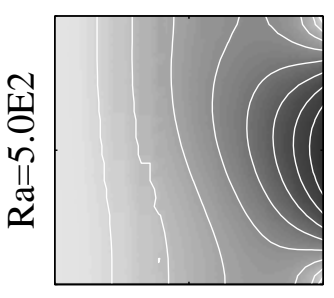

POD SOLUTION

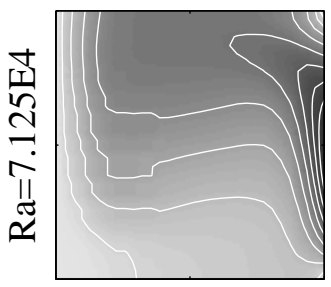

POD SOLUTION

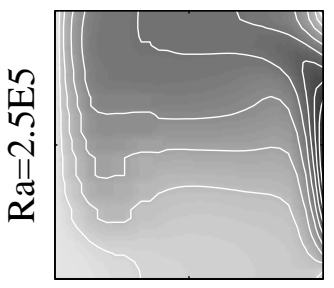

POD SOLUTION

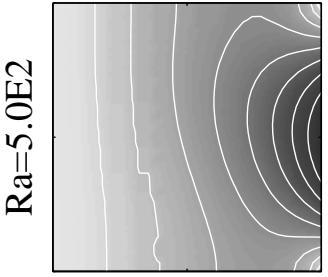

FIDAP SOLUTION

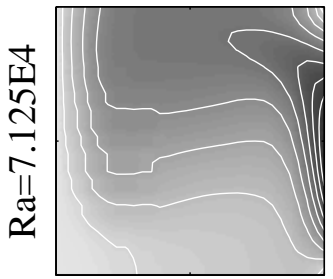

FIDAP SOLUTION

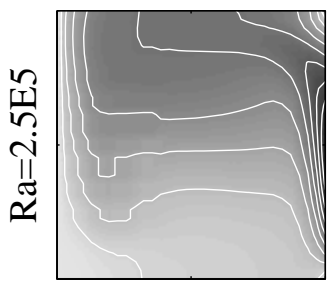

FIDAP SOLUTION

Figure 6: Comparison Between POD and FIDAP Solutions for Rayleigh Numbers Not Belonging to the Ensemble of Snapshots

\section{Boundary Control Applications}

Optimal control problems have been a subject of interest to experimenters and designers for a long time. More recently, flow control has become of substantial interests to mathematicians and computational scientists. Perhaps the most spectacular example of successful flow control is that of aerodynamic control. Here, one determines wing flaps, throttle, etc., so that an aircraft executes a desired maneuver. In this and numerous other examples, the control is computed using either sophisticated mathematical models involving partial differential equations and/or ordinary differential equations or simple empirical rules. In this section, we will demonstrate the effectiveness of using the POD expansion of the ensemble of data to control the temperature distribution inside the cavity without the utilization of a mathematical model. 


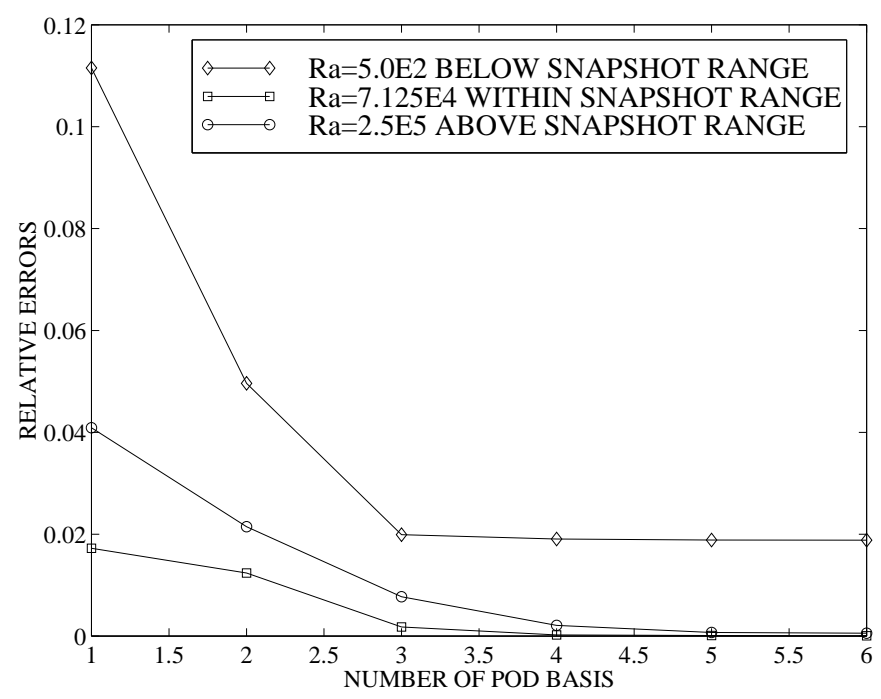

Figure 7: Relative Errors Plotted Against $M$ for Rayleigh Numbers Not Belonging to the Ensemble of Snapshots

\begin{tabular}{|c|c|c|}
\hline Desired Ra & Calculated Ra (using 3 POD modes) & Relative Error \\
\hline 500 & 500 & $1.504 \mathrm{e}-07$ \\
\hline 71,500 & 71,284 & $4.835 \mathrm{e}-04$ \\
\hline 115,000 & 114,939 & $5.317 \mathrm{e}-04$ \\
\hline 250,000 & 249,296 & $2.817 \mathrm{e}-03$ \\
\hline
\end{tabular}

Table 1: Optimal Solutions to the Temperature Tracking Problem

\subsection{Tracking Problem}

Let $T_{d}(\overrightarrow{\mathbf{x}})$ denote a desired temperature distribution inside the cavity. In our case, $T_{d}$ is the numerical solution to the dimensionless steady-state system (3.15)-(3.20) using FIDAP. We want to find the right-wall temperature (or equivalently the Rayleigh number), so that the POD expansion given by

$$
U^{3} \approx \bar{U}+\sum_{k=1}^{3} \alpha_{k}^{R a} \mathbf{\Phi}_{k},
$$

is "close" to $T_{d}$. This boundary control problem can be formulated as finding the Rayleigh number, $R a$, to minimize the cost functional

$$
J(R a)=\int_{\Omega}\left|U^{3}-T_{d}\right|^{2} d \overrightarrow{\mathbf{x}},
$$

where $U^{3}$ is given by equation (5.1) and $\Omega$ is the cavity domain. This unconstrained optimization problem is solved using a combination of golden section search and parabolic interpolation. The results are tabulated in table 1. Here, we are tracking four different temperature profiles corresponding to Rayleigh numbers equal to 500, 71,500, 115,00 and 250,000.

\subsection{Avoiding Hot Spots Problem}

In many applications including the fabrication of microelectronic circuits, it is desirable to avoid "hot spots" along bounding surfaces, i.e., places where the temperature peaks might occur (see, 
e.g., [28]). This is necessary in order to avoid melt-down or structural failures. In our case, we want to find the right wall temperature so that the temperature inside the shaded region in Figure 8 is not greater than the dimensionless temperature 0.1 . That is, we find the temperature on the right wall (equivalently the Rayleigh number) so that to minimize the objective

$$
J(R a)=\sum_{i=1}^{N_{s}} \max \left\{U_{3}^{R a}\left(\overrightarrow{\mathbf{x}}_{i}\right)-0.1,0\right\}+\frac{R a}{10^{4}},
$$

where $U_{3}^{R a}\left(\overrightarrow{\mathbf{x}}_{i}\right)$ are the values of the temperature of the computed solution using 3 POD mode at the nodal points $\overrightarrow{\mathbf{x}}_{i}$, which is inside the shaded region in Figure 8. $N_{s}=120$ is the number of nodal points inside the shaded region. The term $\frac{R a}{10^{4}}$ is included in the cost to achieve the balance between the desired objective and the expended energy to obtain the goal. The results are plotted in Figure 9. The optimal value of the Rayleigh number is 8046.6893 and is compared against two non-optimal solutions. We note that in the non-optimal solution corresponding to $R a=3056.6893$ the right hand corner (darker shade) is above the dimensionless temperature 0.1 . At the other extreme, namely $R a=13046.6893$, the dimensionless temperature is below 0.1 but at the expense of a higher heat input at the right wall.

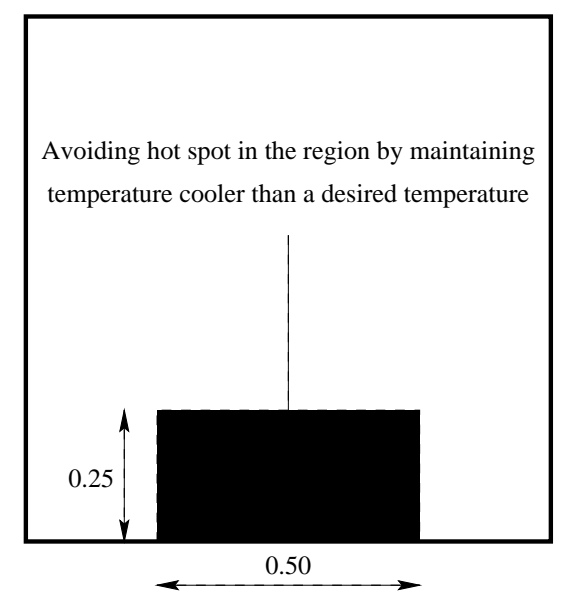

Figure 8: Avoiding Hot Spots Problem Formulation 

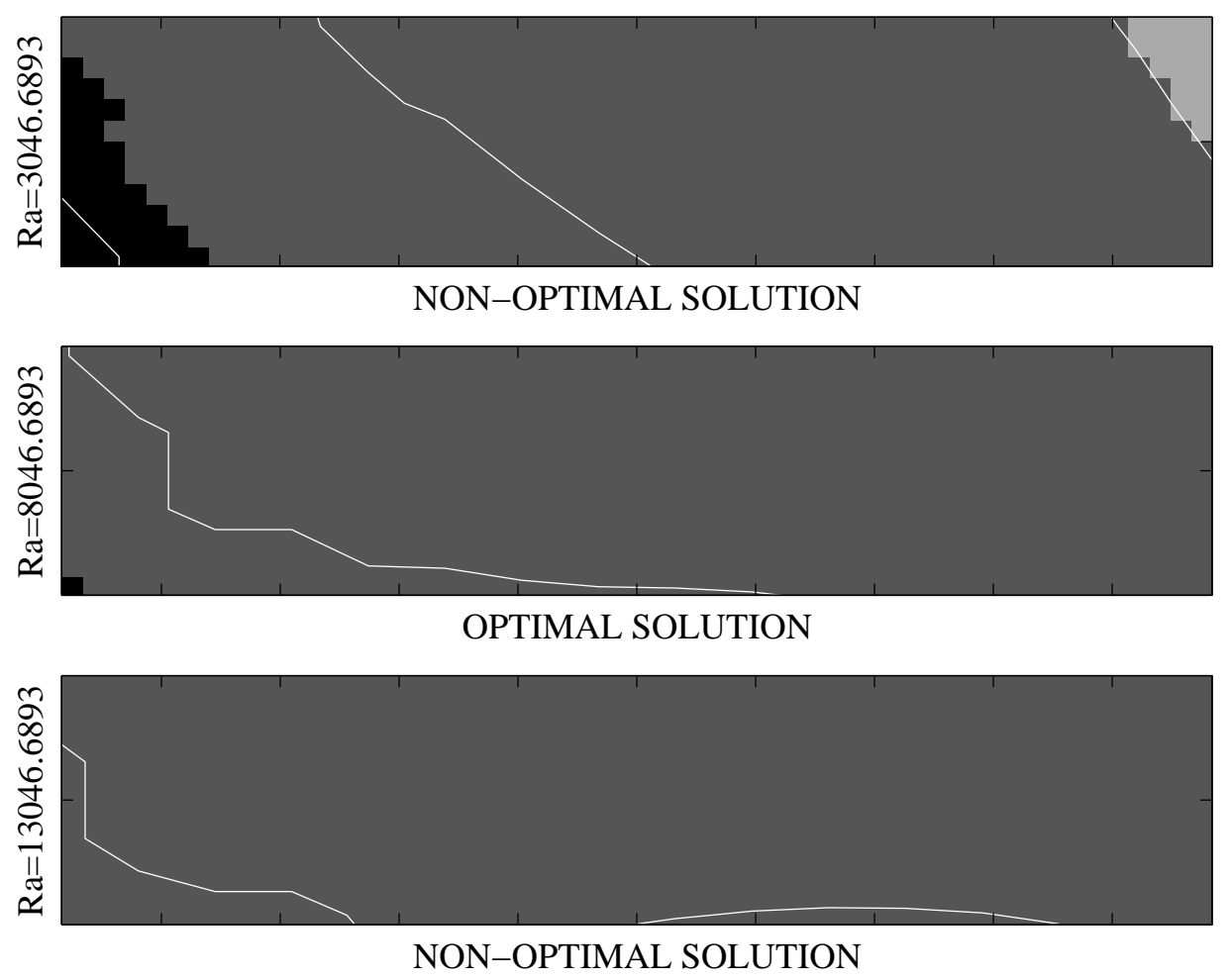

Figure 9: Avoiding Hot Spots Optimal Solution Compared Against Two Non-Optimal Solutions

\section{References}

[1] J.L. Lumley, "The structure of inhomogeneous turbulent flows," in "Atmospheric turbulence and radio wave propagation", A.M. Yaglom and V.I. Tatarski, eds.: Moscow: Nauka (1967) p. 166-178.

[2] N. Aubry, P. Holmes, J.L. Lumley, and E. Stone, "The dynamics of coherent structures in the wall region of a turbulent boundary layer," Journal of Fluid Mechanics, 192 (1988) p. 115-173.

[3] L. Sirovich, "Turbulence and the dynamics of coherent structures, Part II: Symmetries and transformations,", Quart. Appl. Math., XLV (1987) N3:573-582.

[4] G. Berkooz, P. Holmes J.L. Lumley, and J.C. Mattingly, "Low-dimensional models of coherent structures in turbulence," Physics Reports-Review Section of Physics Letters, 287 (1997) N4:338-384.

[5] R. Hilai and J. Rubinstein, "Recognition of rotated images by invariant Karhunen-Loève expansion," Journal of the Optical Society of America A-Optics Image Science and Vision, 11 (1994) N5:1610-1618.

[6] P.J.E. Vermeulen and D.P. Casasent, "Karhunen-Loève techniques for optimal processing of time-sequential imagery,", Optical Engineering, 30 (1991) N4:415-423. 
[7] C.A. Andrews, J.M. Davies, and G.R. Schwarz, "Adaptive data compression,", in Proc. IEEE, 55 (1967), p. 267-277.

[8] S. Watanabe, Karhunen-Loève expansion and factor analysis theoretical remarks and applications," in Proc. 4th Prague Conf. Infom. Theory, 1965.

[9] M. Kirby and L. Sirovich, "Application of the Karhunen-Loève procedure for the characterization of human faces," IEEE Transactions on Pattern Analysis and Machine Intelligence, 12 (1990) N1:103-108.

[10] M. Loève, Probability Theory, Princeton, NJ: van Nostrand (1955).

[11] I.T. Jolliffe, Principle Component Analysis, New York: Springer Verlag (1986).

[12] R.C. Gonzalez and P.A. Wintz, Digital Image Processing, Reading, MA: Addison-Wesley (1987).

[13] K. Pearson, "On lines and planes of closest fit to systems of points in space," Phil. Mag., 6 th series (1901).

[14] H. Hotelling, "Analysis of a complex of statistical variables into principal components," J. Educational Psychol., Sept. (1933).

[15] T. Theodorsen, "Mechanism of Turbulence," in "Proc. 2nd Midwestern Conf. on Fluid Mechanics," Ohio State University, Columbus, OH (1952).

[16] A.A. Townsend, "The Structure of Turbulent Shear Flow," University Press, Cambridge, (1956).

[17] P. Moin and R.D. Moser, "Characteristic-eddy decomposition of turbulence in a channel," Journal Fluids Mechanics, 200 (1989) p. 417-509.

[18] K.S. Ball, L. Sirovich, and L.R. Keefe "Dynamical eigenfunction decomposition of turbulent channel flow," International Journal for Numerical Methods in Fluids, 12 (1991) p. 585-604.

[19] R.S. Reichert, F.F. Hatay, S. Biringer, and A. Husser, "Proper orthogonal decomposition applied to turbulent flows in a square duct," Phys. Fluids Mechanics, 6 (1994) N9:30863092.

[20] M. Rajaee, S.K.F. Karlson, and L. Sirovich, "Low-dimensional description of free-shear-flow coherent structures and their dynamical behavior," Journal of Fluid Mechanics, 258 (1994) p. $1-29$.

[21] M. Kirby, J.P. Boris, and L. Sirovich, "A proper orthogonal decomposition of a simulated supersonic shear layer," International Journal for Numerical Methods in Fluids, 10 (1990) p. 411-428.

[22] D.H. Chambers, R.J. Adrian, P. Moin, D.S. Stewart, and H.J. Sung, "Karhunen-Loève expansion of Burgers' model of turbulence," Phys. Fluids, 31 (1988) p. 2573-2582.

[23] L. Sirovich, "Chaotic dynamics of coherent structures," Physica D, 37 (1989) p. 126-145.

[24] H.V. Ly and H.T. Tran, "Proper orthogonal decomposition for flow calculations and optimal control in a horizontal CVD reactor,", Center for Research in Scientific Computation, North Carolina State University, technical report CRSC-TR98-12 (1998), Quart. Appl. Math., to appear.

[25] H.T. Banks, R.C.H. Del Rosario, and R.C. Smith, "Reduced order model feedback control design: Numerical implementation in a thin shell model," Center for Research in Scientific Computation, North Carolina State University, technical report CRSC-TR98-27 (1998), submitted to IEEE Trans. on AC. 
[26] B. Gebhart, Y. Jaluria, R.L. Mahajan, and B. Sammakia, Buoyancy-Induced Flows and Transport, New York: Hemisphere (1988).

[27] G.N. Facas, Natural convection in a cavity with fins attached to both vertical walls,", in Natural Convection in Enclosures - 1992, (P.G. Simpkins et al. editors), HTD-Vol. 198, ASME.

[28] M.D. Gunzburger, "A prehistory of flow control and optimization," in Flow Control, (M.D. Gunzburger, editor), IMA Volume in Mathematics and Its Applications 68 (1995) p. 185195 . 